\title{
The chiral ring of gauge theories in eight dimensions
}

\section{F. Fucito, ${ }^{a}$ J.F. Morales ${ }^{a}$ and R. Poghossian ${ }^{b}$}

${ }^{a}$ I.N.F.N - sezione di Roma 2, and Università di Roma Tor Vergata, Dipartimento di Fisica, via della Ricerca Scientifica, I-00133 Roma, Italy

${ }^{b}$ Yerevan Physics Institute, Alikhanian Br. 2, AM-0036 Yerevan, Armenia

E-mail: fucito@roma2.infn.it, morales@roma2.infn.it, poghos@yerphi.am

ABSTRACT: We study the non-perturbative corrections generated by exotic instantons in $\mathrm{U}(\mathrm{N})$ gauge theories in eight and four dimensions. As it was shown previously, the eight-dimensional prepotential can be resummed using a plethystic formula showing only a dependence from the center of mass and from a U(1) gauge factor. On the contrary, chiral correlators in eight and four dimensions display a non-trivial dependence from the full gauge group. Furthermore the resolvent, the generating function for the eight and four dimensional correlators, can be written in a compact form both in the eight and four dimensional cases.

Keywords: Brane Dynamics in Gauge Theories, Nonperturbative Effects, Solitons Monopoles and Instantons

ArXiv ePrint: 2010.10235 


\section{Contents}

1 Introduction 1

2 The eight dimensional theory 2

2.1 D(-1)-D7 system on $R^{8}$

$\begin{array}{lll}2.2 & \text { Chiral correlators } & 7\end{array}$

3 The $\mathrm{D}(-1) \mathrm{D} 7$ system on $\mathbb{R}^{4} \times \mathbb{R}^{4} / \mathbb{Z}_{2} \quad 8$

$\begin{array}{lll}3.1 & \text { Chiral correlators } & 10\end{array}$

A Transformation properties of the function $D_{s}(\tau) \quad 11$

\section{Introduction}

In recent years the study of $\mathrm{D} p$ - $\mathrm{D} p^{\prime}$ systems has been particularly fruitful. Such systems can form bound states and preserve part of the original supersymmetry: they are BPS states. Of particular interest is the $\mathrm{D} p-\mathrm{D}(p+4)$ system which accounts for non perturbative effects in four dimensional theories reproducing the ADHM construction for the moduli space of four dimensional gauge instantons [1-6]. In the case in which eight supersymmetric charges are conserved, the prepotential of the four-dimensional gauge theory is codified in the free energy of the statistical partition function counting the low energy excitations of open strings connecting the various D-branes.

The computations of the partition function and of the chiral ring of these theories can be carried out using localization.

Gauge theories with gauge groups $\mathrm{U}(N)$ are realised in term of oriented open strings while $\mathrm{SO}(N)$ and $\mathrm{Sp}(N)$ gauge groups arise when the brane system is on top of an orientifold plane $\mathrm{O} p^{\prime}$. These considerations extend to the case in which the two sets of branes have eight directions with different boundary conditions. The spectrum of twisted non oriented open strings in this case exhibits a lack of bosonic moduli associated to the instanton sizes and gauge orientations. As a consequence these systems exhibit prepotentials behaving as an infinite series of exponentially suppressed corrections in the weak coupling with coefficients given by finite polynomials in the Casimirs formed with the expectation values of the scalar fields in the Coulomb branch. This is on the contrary of what happens in the four dimensional case where, in the weak coupling limit, the coefficients of the prepotential series are given by the inverses of the above mentioned Casimirs. These non perturbative effects, triggered by point like branes dubbed as "exotic instantons", are of great phenomenological interest for their ability to generate Majorana mass terms or Yukawa couplings in four dimensional theories obtained from the eight dimensional theory by dimensional reduction and orbifolding. The four-dimensional counterpart of these exotic 
instantons have been extensively studied with the same motivations [7-14]. Applications to holography in $\mathrm{D}(-1)-\mathrm{D} 3-\mathrm{D} 7$ systems has been considered in $[15,16]$.

Recently the oriented version of the $\mathrm{D}(-1)$-D7 system (or equivalently D0-D8) plus matter has been studied from another perspective $[17,18]$. In absence of the orientifold plane, this system can be made stable by turning on fluxes on the D7-branes so that the ground state of the NS sector comes down to zero mass. The spectrum of massless open strings now has a bosonic degree of freedom and describes the eight-dimensional analog of the ADHM construction of $\mathrm{U}(N)$ instantons [19]. The system can be further decorated by adding $M$ flavour D7 branes with trivial worldvolume fluxes. The gauge partition function for $M=N$ has been computed in $[17,18]$ and written in a compact form using a plethystic resummation. Quite surprisingly the final result is only dependent on an overall $\mathrm{U}(1) \in \mathrm{U}(N) \times \mathrm{U}(M)$ subgroup of the original gauge group. Moreover, the result vanishes in the flat space limit where the $\Omega$ background used by localization is turned off. This means that non perturbative contributions in this theory modify the prepotential only in presence of gravitational fields.

A complete analysis of this brane system from the point of view of string theory will appear in [19]. The aim of this paper is to study the chiral correlators (the chiral ring) of the theory. The results show that unlike the gauge theory prepotential, chiral correlators display a non-trivial dependence on the full gauge group $\mathrm{U}(N)$. Again, non-trivial results require the presence of gravitational fields and can be resummed to all instanton orders in closed form. This is the plan of the paper: in section 2 we recall some basic elements of our construction. In section 3 we compute the resolvent in the eight dimensional case and finally in section 4 we extend our computations to the four dimensional case. In the appendix we collect some results concerning the transformation properties of the eight dimensional resolvent under the $\mathrm{SL}(2, \mathbb{Z})$ group.

\section{The eight dimensional theory}

In this section we review the basic elements of our theory and we introduce the character which allows to compute the prepotential and the chiral correlators generated by exotic instantons in the $\mathrm{U}(N)$ eight-dimensional gauge theories built out of D7-branes with nontrivial worldvolume fluxes.

\section{$2.1 \quad \mathrm{D}(-1)-\mathrm{D} 7$ system on $R^{8}$}

We consider a system of $k \mathrm{D}(-1)$ branes, $N$ D7-branes with a non-trivial worldvolume flux and $M$ flavour D7'-branes (see [19] for details on the brane construction of the system). The instanton moduli arise from the open strings connecting the various branes and with at least one end on the $\mathrm{D}(-1)$-branes. The moduli and the effective action of the theory coming from the open strings starting and ending on $k \mathrm{D}(-1)$ branes can be obtained by reducing to zero dimension the $\mathrm{U}(k)$ gauge theory (SYM) with $N=1$ supersymmetry in ten dimensions. The bosonic field content includes four complex scalars $B_{\ell}, \ell=1,2,3,4$ coming from the gauge vector components along the D7, and a complex field $\chi$ from the transverse components. The 32 components of the spinor in ten dimensions are reduced to 16 because 
our system is $1 / 2$ BPS. In turn these sixteen components, which from the point of view of group theory are in the representations $8_{s}+8_{c}$, will give the supersymmetric partners of the $B_{i}$ and a fermionic lagrangian multiplier to implement the fermionic constraint on the moduli. Under SU(4) we will get $8_{s}=4 \oplus \overline{4}$ and $8_{s}=6 \oplus 1 \oplus 1$ so we will describe these degrees of freedom as four complex fermions $M_{\ell}$, the partners of $B_{\ell}$, a component $\lambda_{-}$paired under supersymmetry with $\bar{\chi}$ and seven real components $\left\{\lambda_{[I J]}, \lambda^{[I J]}, \lambda_{+}\right\}$with $I, J=1,2,3$ and $I<J$ paired with the seven auxiliary fields $\left\{D_{[I J]}, D^{[I J]}, D_{7}\right\}$ implementing the vacuum conditions. All $\mathrm{D}(-1)-\mathrm{D}(-1)$ fields transform in the adjoint of the $\mathrm{U}(k)$ symmetry group. On the other hand the $\mathrm{D}(-1)-\mathrm{D} 7$ and $\mathrm{D}(-1)$-D7' strings provide the moduli $(w, \mu)$ and $\left(\mu^{\prime}, h\right)$ in the $\mathrm{U}(N) \times \mathrm{U}(k)$ and $\mathrm{U}(M) \times \mathrm{U}(k)$ bifundamental representations respectively.

To localize the partition function we have to make the theory equivariant with respect to the Cartan of the group $G=\mathrm{U}(k) \otimes \mathrm{U}(N) \otimes \mathrm{U}(M) \otimes \mathrm{SO}(8)$ where $\mathrm{U}(k), \mathrm{U}(N), \mathrm{U}(M)$ and $\mathrm{SO}(8)$ are the instanton, gauge, flavour and eight-dimensional Lorentz groups respectively. We parametrize the Cartan subgroups by $\chi=\operatorname{diag}\left\{\chi_{i}\right\}, a=\operatorname{diag}\left\{a_{u}\right\}, m=\operatorname{diag}\left\{m_{s}\right\}$ and $\epsilon_{\ell}$ respectively with

$$
\epsilon_{1}+\epsilon_{2}+\epsilon_{3}+\epsilon_{4}=0
$$

given that the $\mathrm{SO}(8)$ is broken to $\mathrm{SO}(7)$ since there are seven ADHM constraints for our system. The diagonal matrices $\chi, a$ and $m$ parametrize the positions of the $\mathrm{D}(-1), \mathrm{D} 7$ and D7' branes respectively along the direction transverse to the D7-branes. The $a_{u}$ 's describe the vev's of the scalar fields and the $\epsilon_{\ell}$ 's the eight-dimensional $\Omega$-background.

The equivariantly deformed supersymmetric variation $\mathcal{Q}$ reads

$$
\begin{aligned}
\mathcal{Q} B_{\ell} & =M_{\ell}, & \mathcal{Q} M_{\ell} & =\left[\chi, B_{\ell}\right]+\epsilon_{\ell} B_{\ell} \\
\mathcal{Q} \lambda^{I J} & =D^{I J}, & \mathcal{Q} D^{I J} & =\left[\chi, \lambda^{I J}\right]+\left(\epsilon_{I}+\epsilon_{J}\right) \lambda^{I J} \\
\mathcal{Q} \lambda_{+} & =D_{7}, & \mathcal{Q} D_{7} & =\left[\chi, \lambda_{+}\right] \\
\mathcal{Q} \bar{\chi} & =\lambda_{-}, & \mathcal{Q} \lambda_{-} & =[\chi, \bar{\chi}] \\
\mathcal{Q} w & =\mu, & \mathcal{Q} \mu & =\chi w+w a \\
\mathcal{Q} \mu^{\prime} & =h, & \mathcal{Q} h & =\chi \mu^{\prime}+\mu^{\prime} M \\
\mathcal{Q} \chi & =0 & &
\end{aligned}
$$

The field content and the transformation properties with respect to $\mathrm{U}(k) \times \mathrm{U}(N) \times \mathrm{U}(M) \times \mathrm{SO}(8)$ for the eight dimensional case are summarized in table 1. We find it convenient to compute the $\mathcal{Q}^{2}$ eigenvalues of the various fields by introducing the following character

$$
\chi=\operatorname{tr}_{\mathcal{H}} e^{-\beta \mathcal{Q}^{2}}=-\sum_{i<j=1}^{k} \frac{x_{i}}{x_{j}} \prod_{\ell=1}^{4}\left(1-T_{\ell}\right)-\sum_{i=1}^{k} \prod_{I=1}^{3}\left(1-T_{I}\right)+\sum_{i=1}^{k}\left(\sum_{u=1}^{N} \frac{T_{a_{u}}}{x_{i}}-\sum_{f=1}^{M} \frac{T_{m_{f}}}{x_{i}}\right)
$$

where

$$
T_{\ell}=e^{-\beta \epsilon_{\ell}} \quad, \quad x_{i}=e^{-\beta \chi_{i}} \quad, \quad T_{a_{u}}=e^{-\beta a_{u}} \quad, \quad T_{m_{f}}=e^{-\beta m_{f}}
$$

According to (2.1) one finds that the $T_{\ell}$ 's satisfy the relation

$$
T_{1} T_{2} T_{3} T_{4}=1
$$




\begin{tabular}{|c|c|c|c|}
\hline$(\Phi, \mathcal{Q} \Phi)$ & $(-)^{F}$ & $\mathrm{U}(k) \times \mathrm{U}(N) \times \mathrm{U}(M)$ & $\mathcal{Q}^{2}$ \\
\hline$\left(B_{\ell}, M_{\ell}\right)$ & + & $(\mathbf{k} \overline{\mathbf{k}}, \mathbb{1}, \mathbb{1})$ & $T_{\ell} \frac{x_{i}}{x_{j}}$ \\
$\left(\bar{B}_{\ell}, M^{\ell}\right)$ & + & $(\mathbf{k} \overline{\mathbf{k}}, \mathbb{1}, \mathbb{1})$ & $T_{\ell}^{-1} \frac{x_{i}}{x_{j}}$ \\
$\left(\lambda^{I J}, D^{I J}\right)$ & - & $(\mathbf{k} \overline{\mathbf{k}}, \mathbb{1}, \mathbb{1})$ & $T_{I} T_{J} \frac{x_{i}}{x_{j}}$ \\
$\left(\lambda_{I J}, D_{I J}\right)$ & - & $(\mathbf{k} \overline{\mathbf{k}}, \mathbb{1}, \mathbb{1})$ & $T_{I}^{-1} T_{J}^{-1} \frac{x_{i}}{x_{j}}$ \\
$\left(\lambda_{+}, D_{7}\right)$ & - & $(\mathbf{k} \overline{\mathbf{k}}, \mathbb{1}, \mathbb{1})$ & $\frac{x_{i}}{x_{j}}$ \\
$\left(\bar{\chi}, \lambda_{-}\right)$ & + & $(\mathbf{k} \overline{\mathbf{k}}, \mathbb{1}, \mathbb{1})$ & $\frac{x_{i}}{x_{j}}$ \\
$(w, \mu)$ & + & $(\overline{\mathbf{k}}, \mathbf{N}, \mathbb{1})$ & $\frac{T_{a_{u}}}{x_{i}}$ \\
$\left(\mu^{\prime}, h^{\prime}\right)$ & - & $(\overline{\mathbf{k}}, \mathbb{1}, \mathbf{M})$ & $\frac{T_{m_{f}}}{x_{i}}$ \\
\hline
\end{tabular}

Table 1. Instanton moduli space. The domain of the indices for $\mathbb{R}^{8}$ are: $\ell=1, \ldots 4, I, J=1, \ldots 3$ with $I<J, i, j=1, \ldots, k, u=1, \ldots, N$ and $f=1, \ldots, M$.

The character (2.3) provides the natural generalization of the four dimensional character which, as detailed in [20], keeps into account the contributions of the various degrees of freedom. Each monomial in (2.3) represents a supermultiplet in table 1 contributing with plus or minus depending on whether the multiplet involve a physical or an auxiliary field respectively. In particular the linear and cubic terms in $T_{\ell}$ in the expression $\prod_{\ell=1}^{4}\left(1-T_{\ell}\right)$ represent the contributions of $B_{\ell}, \bar{B}^{\ell}$, while the quadratic ones subtract the degrees of freedom associated to the ADHM constraints $\left(D_{I J}\right.$ and $\left.\bar{D}^{I J}\right)$. The novelty with respect to the four dimensional case is that, due to the constraint $(2.5)$, the term $\prod_{\ell=1}^{4}\left(1-T_{\ell}\right)$ in the eight dimensional character contains not only the degrees of freedom of the fields but also the contribution of their complex conjugates thus doubling the degrees of freedom. To restore the right counting of degrees of freedom we restrict the sum over $i, j$ to $i<j$. The second term in (2.3) subtracts half the degrees of freedom of the diagonal $i=j$ terms. Finally the last two terms in (2.3) account for the open strings ending on D7 and D7' branes.

After localizing and computing the $\operatorname{determinant} \operatorname{det} \mathcal{Q}^{2}$, the partition function is given by $Z_{9 d}=\sum_{k} Z_{k} q^{k}$ with $^{1}$

$$
\begin{aligned}
Z_{k}= & \frac{\mathcal{E}^{k}}{k !} \int \prod_{i=1}^{k} \frac{d x_{i}}{x_{i}} \prod_{i<j}^{k} \frac{\left(1-\frac{x_{i}}{x_{j}}\right)\left(1-\frac{x_{i}}{x_{j}} T_{1} T_{2}\right)\left(1-\frac{x_{i}}{x_{j}} T_{1} T_{3}\right)\left(1-\frac{x_{i}}{x_{j}} T_{2} T_{3}\right)}{\left(1-\frac{x_{i}}{x_{j}} T_{1}\right)\left(1-\frac{x_{i}}{x_{j}} T_{2}\right)\left(1-\frac{x_{i}}{x_{j}} T_{3}\right)\left(1-\frac{x_{i}}{x_{j}} T_{4}\right)} \\
& \times \prod_{i=1}^{k} \frac{\prod_{f=1}^{M}\left(1-\frac{T_{M_{f}}}{x_{i}}\right)}{\prod_{u=1}^{N}\left(1-\frac{T_{a_{u}}}{x_{i}}\right)}
\end{aligned}
$$

\footnotetext{
${ }^{1}$ The terms appearing in the integrand of (2.6) come straighforwardly from the character (2.3). Each term $\bullet$ in the character contributes a $(1-\bullet)^{ \pm}$with \pm depending on the sign of the monomial in the character.
} 
with

$$
\mathcal{E}=\frac{\left(1-T_{1} T_{2}\right)\left(1-T_{1} T_{3}\right)\left(1-T_{2} T_{3}\right)}{\left(1-T_{1}\right)\left(1-T_{2}\right)\left(1-T_{3}\right)\left(1-T_{4}\right)}
$$

We refer to $Z_{9 \mathrm{~d}}$ as the nine dimensional partition function, resulting from the lift of the eight dimensional theory to $\mathbb{R}^{8} \times S^{1}$ along a circle of radius $\beta$. The case $N=M$ was the one considered in [18]. Formula (2.6) has some sign differences from the ones appearing in [18] where these signs have been fixed order by order to produce the plethystic result displayed below. We have checked that the partition function computed using (2.6) reproduces the plethystic exponential up to $k=15$ without any sign addition.

The poles of the integrand in (2.6) can be put in correspondence with sets of $N$ solid partitions (four dimensional Young diagrams) with $k$ total number of boxes (instanton number). Imposing a lexicographic order we numerate the boxes of the set $\left\{Y_{u}\right\}$ by a single integer label $i$, each associated to a box with coordinates $\left(m_{1}, m_{2}, m_{3}, m_{4}\right) \in Y_{u}$ and coordinate $x_{i}^{Y}$ given by

$$
\sum_{i=1}^{k} x_{i}^{Y}=\sum_{u=1}^{N} \sum_{\left\{m_{\ell}\right\} \in Y_{u}}^{k} T_{a_{u}} T_{1}^{m_{1}-1} T_{2}^{m_{2}-1} T_{3}^{m_{3}-1} T_{4}^{m_{4}-1}
$$

The partition function is given by the sum $Z_{9 d}=\sum_{Y} Z_{9 Y} q^{|Y|}$ over the $N$ solid partitions $\left\{Y_{u}\right\}$ of the residues of the integrand in (2.6) at $x_{i}=x_{i}^{Y}$. For instance for $k=1$ one finds, $\mathrm{N}$ diagrams contributing, each with a single box centered at $a_{u}$. If $N=M$ after summing over the $N$ contributions one finds

$$
Z_{1}=\frac{\left(1-T_{1} T_{2}\right)\left(1-T_{1} T_{3}\right)\left(1-T_{2} T_{3}\right)\left(1-T_{\mu}\right)}{\left(1-T_{1}\right)\left(1-T_{2}\right)\left(1-T_{3}\right)\left(1-T_{4}\right)}
$$

with $T_{\mu}=e^{-\beta \mu}$ and

$$
\mu=\sum_{u=1}^{N}\left(m_{u}-a_{u}\right)
$$

Similarly for higher $k$, the partition function can be computed. Remarkably, the all instanton result can be written in the simple plethystic form [18]

$$
Z_{9 \mathrm{~d}}=\exp \left[\sum_{n=1}^{\infty} \frac{f\left(T_{\ell}^{n}, T_{\mu}^{n}, q^{n}\right)}{n}\right]
$$

where, as usual, $q=e^{2 \pi i \tau}$. $\tau$ is the complex coupling of our gauge theory and

$$
f\left(T_{\ell}, T_{\mu}, q\right)=\frac{\left(1-T_{1} T_{2}\right)\left(1-T_{1} T_{3}\right)\left(1-T_{2} T_{3}\right) q}{\left(1-T_{1}\right)\left(1-T_{2}\right)\left(1-T_{3}\right)\left(1-T_{1} T_{2} T_{3}\right)} \frac{\left(1-T_{\mu}\right)}{\left(1-q T_{\mu}\right)(1-q)}
$$

Remarkably, the results either depend only on the specific combination $\mu$ involving the total mass and the overall center of mass $\mathrm{U}(1)$. The results for $M<N$ can be obtained by sending $\mu \rightarrow \infty$, leading again to the plethystic formulas $(2.11)-(2.12)$ with $T_{\mu}=0$. Remarkably all dependence on the remaining masses and expectation values cancel in this limit. 
The eight dimensional partition function is obtained from $Z_{9 d}$ by sending $\beta$ to zero

$$
Z_{8 \mathrm{~d}}\left(a, m, \epsilon_{\ell}, q\right)=\lim _{\beta \rightarrow 0} Z_{9 \mathrm{~d}}\left(T_{a}, T_{m}, q\right)
$$

Again the partition function can be written as a sum over $4 \mathrm{~d}$ solid partitions $\left\{Y_{u}\right\}$

$$
\begin{aligned}
Z_{8 \mathrm{~d}}\left(a, m, \epsilon_{\ell}, q\right)= & \sum_{k} q^{k} Z_{8 k}=\sum_{Y} q^{|Y|} Z_{8 Y} \\
Z_{8 k}= & \frac{\mathcal{V}^{k}}{k !} \int \prod_{i=1}^{k} d \chi_{i} \prod_{i<j}^{k} \frac{\chi_{j i}\left(\chi_{j i}+\epsilon_{1}+\epsilon_{2}\right)\left(\chi_{j i}+\epsilon_{1}+\epsilon_{3}\right)\left(\chi_{j i}+\epsilon_{2}+\epsilon_{3}\right)}{\left(\chi_{j i}+\epsilon_{1}\right)\left(\chi_{j i}+\epsilon_{2}\right)\left(\chi_{j i}+\epsilon_{3}\right)\left(\chi_{j i}+\epsilon_{4}\right)} \\
& \times \prod_{i=1}^{k} \frac{\prod_{f=1}^{M}\left(\chi_{i}-m_{f}\right)}{\prod_{u=1}^{N}\left(\chi_{i}-a_{u}\right)}
\end{aligned}
$$

with $\chi_{j i}=\chi_{j}-\chi_{i}$, and

$$
\mathcal{V}=\frac{\left(\epsilon_{1}+\epsilon_{2}\right)\left(\epsilon_{1}+\epsilon_{3}\right)\left(\epsilon_{2}+\epsilon_{3}\right)}{\epsilon_{1} \epsilon_{2} \epsilon_{3} \epsilon_{4}}
$$

The eight-dimensional prepotential is defined as

$$
\mathcal{F}_{8}\left(a, \epsilon_{\ell}, q\right)=\epsilon_{1} \epsilon_{2} \epsilon_{3} \epsilon_{4} \log Z_{8 d}
$$

In the case $M=N$ we get

$$
\begin{aligned}
\mathcal{F}_{8}\left(a, \epsilon_{\ell}, q\right) & =\left(\epsilon_{1}+\epsilon_{2}\right)\left(\epsilon_{1}+\epsilon_{3}\right)\left(\epsilon_{2}+\epsilon_{3}\right) \mu \sum_{n=1}^{\infty} \frac{q^{n}}{n\left(1-q^{n}\right)^{2}} \\
& =\left(\epsilon_{1}+\epsilon_{2}\right)\left(\epsilon_{1}+\epsilon_{3}\right)\left(\epsilon_{2}+\epsilon_{3}\right) \mu \log \mathcal{M}_{3}(q)
\end{aligned}
$$

where

$$
\mathcal{M}_{3}(q)=\prod_{n=1}^{\infty} \frac{1}{\left(1-q^{n}\right)^{n}}
$$

is the Mac Mahon function counting the three dimensional partitions of the integers. The factor $\epsilon_{1} \epsilon_{2} \epsilon_{3} \epsilon_{4}$ gets rid of the superspace volume in eight dimensions $\int d^{8} x d^{8} \theta=1 /\left(\epsilon_{1} \epsilon_{2} \epsilon_{3} \epsilon_{4}\right)$. We notice that formula (2.15) for generic $M$ can be obtained from that for $M=N$ by sending some masses to infinity keeping constant $\hat{q}=q \prod_{f>M}^{N}\left(-m_{f}\right)$. The results for $M<N$ can then be obtained from (2.18) by sending $\mu \rightarrow \infty$ and $q \rightarrow 0$ with $\mu^{N-M} q$ kept fixed. One finds

$$
\mathcal{F}_{8}\left(a, \epsilon_{\ell}, q\right)=\left\{\begin{array}{cc}
\left(\epsilon_{1}+\epsilon_{2}\right)\left(\epsilon_{1}+\epsilon_{3}\right)\left(\epsilon_{2}+\epsilon_{3}\right) \hat{q} & M=N-1 \\
0 & M<N-1
\end{array}\right.
$$

The prepotential $\mathcal{F}_{8}$ defines the non-perturbative effective action of the eight-dimensional theory

$$
S_{\text {eff }}=\int d^{8} x d^{8} \theta \mathcal{F}_{8}(\Phi, W, q)
$$

after the parameters $a$ and $\epsilon_{\ell}$ are promoted to superfields $\Phi, W$. We observe that only the $\mathrm{U}(1)$ part of the prepotential is affected by instanton corrections. We will show below that this is not the case for the correlators of the gauge theory that exhibit a full dependence from the $\mathrm{SU}(N)$ gauge degrees of freedom. 


\subsection{Chiral correlators}

In this section we will focus on the chiral correlators $\left\langle\operatorname{tr} \phi^{J}\right\rangle$ of the gauge theory. According to localization [27], the generating function of these correlators is given by

$$
\left\langle\operatorname{tr} e^{z \phi}\right\rangle=\operatorname{tr} e^{z a}-\frac{1}{Z_{8 d}} \sum_{Y} q^{|Y|} Z_{8 Y} \sum_{i=1}^{k}\left(x_{i}^{Y}\right)^{z} \prod_{\ell=1}^{4}\left(1-T_{\ell}^{z}\right)
$$

$x_{i}^{Y}$ denotes the location of the corresponding pole given by (2.8). We notice that in the eight-dimensional limit $\beta \rightarrow 0$, the right hand side of (2.22) starts as $z^{4}$, so all correlators with $J<4$ receive no instanton corrections. The first non-trivial correlator is then given by the coefficient of the $z^{4}$ term in (2.22)

$$
\left\langle\operatorname{tr} \phi^{4}\right\rangle=\operatorname{tr} a^{4}-\frac{24 \epsilon_{1} \epsilon_{2} \epsilon_{3} \epsilon_{4}}{Z_{8 d}} \sum_{Y} k q^{k} Z_{8 k}=\operatorname{tr} a^{4}-24 q \frac{d}{d q} \mathcal{F}_{8}(q)
$$

This provides the eight-dimensional analog of the relation found in [27]. Similar higher correlators can be evaluated order by order in $q$. We find it convenient to pack the correlators $\left\langle\operatorname{tr} \phi^{J}\right\rangle$ into a different generating function, the so called resolvent, defined by

$$
\left\langle\operatorname{tr} \frac{1}{x-\phi}\right\rangle=\sum_{J=0}^{\infty} \frac{\left\langle\operatorname{tr} \phi^{J}\right\rangle}{x^{J+1}}
$$

This function is closely related to the Seiberg-Witten curve [28] and will be written below in a closed form.

Let us consider first the $\mathrm{U}(1)$ case without mass multiplets, i.e. $N=1, M=0$. Up to order $q^{16}$ one finds

$$
\begin{aligned}
& \left\langle\operatorname{tr} \frac{1}{x-\phi}\right\rangle=\frac{1}{x-a}+12 \epsilon^{(3)}\left(\frac{2 q}{(x-a)^{5}}-\frac{25 q^{2}}{(x-a)^{6}}+\frac{100 q^{3}}{(x-a)^{7}}-\frac{735 q^{4}}{2(x-a)^{8}}\right. \\
& +\frac{728 q^{5}}{(x-a)^{9}}-\frac{2100 q^{6}}{(x-a)^{10}}+\frac{3000 q^{7}}{(x-a)^{11}}-\frac{14025 q^{8}}{2(x-a)^{12}}+\frac{10010 q^{9}}{(x-a)^{13}}-\frac{18590 q^{10}}{(x-a)^{14}} \\
& \left.+\frac{22204 q^{11}}{(x-a)^{15}}-\frac{47775 q^{12}}{(x-a)^{16}}+\frac{47600 q^{13}}{(x-a)^{17}}-\frac{85000 q^{14}}{(x-a)^{18}}+\frac{106080 q^{15}}{(x-a)^{19}}-\frac{330429 q^{16}}{2(x-a)^{20}}+\ldots\right)+O\left(\epsilon^{4}\right)
\end{aligned}
$$

with

$$
\epsilon^{(3)} \equiv\left(\epsilon_{1}+\epsilon_{2}\right)\left(\epsilon_{1}+\epsilon_{3}\right)\left(\epsilon_{2}+\epsilon_{3}\right) .
$$

After some educated guessing the full answer can be written in closed form as

$$
\left\langle\operatorname{tr} \frac{1}{x-\phi}\right\rangle=\frac{1}{x-a}-\epsilon^{(3)} \frac{\partial^{3}}{\partial x^{3}}\left[\frac{1}{a-x} D_{3}\left(\frac{q}{a-x}\right)\right]+O\left(\epsilon^{4}\right)
$$

where we introduce the function

$$
D_{3}(q)=\sum_{n=1}^{\infty} \sigma_{2}(n) q^{n}=\sum_{k=1}^{\infty} \frac{k^{2} q^{k}}{1-q^{k}}=q \frac{d}{d q} \log \mathcal{M}_{3}(q)
$$


with

$$
\sigma_{2}(n)=\sum_{d \mid n} d^{2}
$$

the sum of all squared divisors $d^{2}$ of $n$. The generalization to $\mathrm{U}(N)$ with flavour group $\mathrm{U}(M)$ is straightforward. One finds

$$
\left\langle\operatorname{tr} \frac{1}{x-\phi}\right\rangle=\frac{P^{\prime}(x)}{P(x)}-\epsilon^{(3)} \frac{\partial^{3}}{\partial x^{3}}\left[\frac{R^{\prime}(x)}{R(x)} D_{3}(q R(x))+O(\epsilon)\right]
$$

with

$$
P(x)=\prod_{u=1}^{N}\left(x-a_{u}\right) \quad, \quad R(x)=\frac{\prod_{f=1}^{M}\left(m_{u}-x\right)}{\prod_{u=1}^{N}\left(a_{u}-x\right)}
$$

Once again we notice that, as in the case of the prepotential, these correlators get corrected only in the presence of gravitational fields given the presence of the factor (2.26), but now non-trivial instanton corrections modify also the $\mathrm{SU}(N)$ part of the theory. For instance, specifying to $\mathrm{SU}(2)$ theory with two flavours, and taking $a_{1}=-a_{2}=a, m_{1}=-m_{2}=m$, one finds for the first non-trivial correlators

$$
\begin{aligned}
& \left\langle\operatorname{tr} \phi^{5}\right\rangle=120 \epsilon^{(3)}\left(m^{2}-a^{2}\right)\left(q+5 q^{2}+10 q^{2}+\ldots\right) \\
& \left\langle\operatorname{tr} \phi^{7}\right\rangle=420 \epsilon^{(3)}\left(m^{2}-a^{2}\right)\left(2 q+5\left(3 a^{2}-m^{2}\right) q^{2}+20\left(2 a^{2}-m^{2}\right) q^{3}+\ldots\right)
\end{aligned}
$$

and so on. We conclude this section observing that the infinite series $D_{3}(\tau)$ appearing in the correlators differs from an Eisenstein series because the power of $k$ in (2.28) is even rather than odd. This difference modifies drastically the behaviour of the function under $\operatorname{SL}(2, \mathbb{Z})$ transformations of its gauge coupling $\tau$. We find (see appendix for details)

$$
\begin{aligned}
D_{3}\left(\frac{a \tau+b}{c \tau+d}\right)= & (c \tau+d)^{3} D_{3}(\tau) \\
& +\frac{i}{4 \pi^{3}}\left(\left(1+(c \tau+d)^{3}\right) \zeta(3)+2 \sum_{m=1}^{\infty} \sum_{n=1}^{\infty} \frac{(c \tau+d)^{3}}{((c \tau+d) m+n)^{3}}\right)
\end{aligned}
$$

showing the obstruction to modularity.

\section{The $\mathrm{D}(-1) \mathrm{D} 7$ system on $\mathbb{R}^{4} \times \mathbb{R}^{4} / \mathbb{Z}_{2}$}

As we stated in the introduction the eight dimensional case is a clean example in which the non perturbative effects due to what we called exotic instantons are displayed. Nonetheless, possible phenomenological applications should take place in our four dimensional world. It is possible to compute such effects wrapping $\mathrm{N}$ fractional D7 and M fractional D7' branes on $\mathbb{R}^{4} / \mathbb{Z}_{2}$ and focusing on the exotic prepotentials generated by the $\mathrm{D}(-1)$-instantons. The prepotential obtained in such a way defines a four dimensional effective action

$$
S_{\text {eff }}=\int d^{4} x d^{4} \theta \mathcal{F}(\Phi, W)
$$

where $\Phi, W$ are the superfields of which the scalar field and the $\Omega$ background are one of the components. The form (3.1) is dictated by supersymmetry, the important difference 


\begin{tabular}{|c|c|c|c|}
\hline$(\Phi, \mathcal{Q} \Phi)$ & $(-)^{F}$ & $\mathrm{U}(k) \times \mathrm{U}(N) \times \mathrm{U}(M)$ & $\mathcal{Q}^{2}$ \\
\hline$\left(B_{\ell}, M_{\ell}\right)$ & + & $(\mathbf{k} \overline{\mathbf{k}}, \mathbb{1}, \mathbb{1})$ & $T_{\ell} \frac{x_{i}}{x_{j}}$ \\
$\left(\lambda^{I J}, D^{I J}\right)$ & - & $(\mathbf{k} \overline{\mathbf{k}}, \mathbb{1}, \mathbb{1})$ & $T_{I} T_{J} \frac{x_{i}}{x_{j}}$ \\
$\left(\lambda_{+}, D_{7}\right)$ & - & $(\mathbf{k} \overline{\mathbf{k}}, \mathbb{1}, \mathbb{1})$ & $\frac{x_{i}}{x_{j}}$ \\
$\left(\bar{\chi}, \lambda_{-}\right)$ & + & $(\mathbf{k} \overline{\mathbf{k}}, \mathbb{1}, \mathbb{1})$ & $\frac{x_{i}}{x_{j}}$ \\
$(w, \mu)$ & + & $(\overline{\mathbf{k}}, \mathbf{N}, \mathbb{1})$ & $\frac{T_{a}}{x_{i}}$ \\
$\left(\mu^{\prime}, h^{\prime}\right)$ & - & $(\overline{\mathbf{k}}, \mathbb{1}, \mathbf{M})$ & $\frac{T_{m}}{x_{i}}$ \\
\hline
\end{tabular}

Table 2. Instanton moduli space. The domain of the indices for $\mathbb{R}^{4} \times \mathbb{R}^{4} / \mathbb{Z}_{2}$ are: $\ell, I, J=1,2$ with $I<J, i, j=1, \ldots, k, u=1, \ldots, N$ and $f=1, \ldots, M$.

with the standard case is that prepotentials generated by exotic instantons are polynomials in the vev. These effects are purely stringy, generated by wrapped euclidean branes along the internal space and not present in "standard" field theory. The moduli content of the exotic theory is given in table 2. With respect to the eight dimensional case, the fields labeled by the indices $\ell=3,4$ and $I, J=3$ are projected out by the orbifold quotient. The character reduces to

$$
\chi=\operatorname{tr}_{\mathcal{H}} e^{-\beta \mathcal{Q}^{2}}=-\sum_{i, j=1}^{k} \frac{x_{i}}{x_{j}}\left(1-T_{1}\right)\left(1-T_{2}\right)+\sum_{i=1}^{k}\left(\sum_{u=1}^{N} \frac{T_{a_{u}}}{x_{i}}-\sum_{f=1}^{M} \frac{T_{m_{f}}}{x_{i}}\right)
$$

With respect to the nine dimensional character (2.3) there is no need for a condition of the type $i<j$ given that a condition of the type of (2.5) is absent and the "volume" $\left(1-T_{1}\right)\left(1-T_{2}\right)$ correctly contains the four terms corresponding to the degrees of freedom of the fields $B_{1}, B_{2}$ and the constraints due to the $\mathrm{U}(k)$ symmetry and the complex ADHM constraint. Following the same procedure of the previous section we find

$$
Z_{k}=\frac{\mathcal{E}^{k}}{k !} \int \prod_{i=1}^{k} \frac{d x_{i}}{x_{i}} \prod_{i \neq j}^{k} \frac{\left(1-\frac{x_{i}}{x_{j}}\right)\left(1-\frac{x_{i}}{x_{j}} T_{1} T_{2}\right)}{\left(1-\frac{x_{i}}{x_{j}} T_{1}\right)\left(1-\frac{x_{i}}{x_{j}} T_{2}\right)} \prod_{i=1}^{k} \frac{\prod_{f=1}^{M}\left(1-\frac{T_{M_{f}}}{x_{i}}\right)}{\prod_{u=1}^{N}\left(1-\frac{T_{a_{u}}}{x_{i}}\right)}
$$

with

$$
\mathcal{E}=\frac{\left(1-T_{1} T_{2}\right)}{\left(1-T_{1}\right)\left(1-T_{2}\right)}
$$

The partition function can now be written in the plethystic form

$$
Z_{5 d}=\exp \left[\sum_{n=1}^{\infty} \frac{f^{\prime}\left(T_{\ell}^{n}, T_{\mu}^{n}, q^{n}\right)}{n}\right]
$$


with

$$
\begin{array}{lll}
f^{\prime}\left(T_{\ell}, T_{\mu}, q\right)=\frac{\left(1-T_{1} T_{2}\right)\left(1-T_{\mu}\right) q}{\left(1-T_{1}\right)\left(1-T_{2}\right)} & \text { if } & M=N \\
f^{\prime}\left(T_{\ell}, T_{\mu}, q\right)=\frac{\left(1-T_{1} T_{2}\right) q}{\left(1-T_{1}\right)\left(1-T_{2}\right)} & \text { if } & M=N-1 \\
f^{\prime}\left(T_{\ell}, T_{\mu}, q\right)=0 & \text { if } & M \leq N-2
\end{array}
$$

Evaluating the first few instanton orders and sending $\beta, \epsilon_{\ell} \rightarrow 0$, one finds that the four dimensional prepotential resums to the simple form

$$
\begin{array}{lll}
\mathcal{F}_{4}=-\mu\left(\epsilon_{1}+\epsilon_{2}\right) \log (1-q) & \text { if } & M=N \\
\mathcal{F}_{4}=\left(\epsilon_{1}+\epsilon_{2}\right) q & \text { if } & M=N-1 \\
\mathcal{F}_{4}=0 & \text { if } & M \leq N-2
\end{array}
$$

with $\mu$ given again by (2.10). Also in this four dimensional case we find that only the $\mathrm{U}(1)$ gauge degrees of freedom correct the classical prepotential.

\subsection{Chiral correlators}

Chiral correlators $\left\langle\operatorname{tr} \phi^{J}\right\rangle$ in the four-dimensional gauge theory are computed now by

$$
\left\langle\operatorname{tr} e^{z \phi}\right\rangle=\operatorname{tr} e^{z a}-\frac{1}{Z_{4 d}} \sum_{Y} q^{|Y|} Z_{4 Y} \sum_{i=1}^{k}\left(x_{i}^{Y}\right)^{z}\left(1-T_{1}^{z}\right)\left(1-T_{2}^{z}\right)
$$

with $Z_{Y}$ the contribution to the partition function of the solid partition set $Y=\left\{Y_{u}\right\}$ and $x_{i}^{Y}$ denotes the location of the corresponding pole given by (2.8). We notice that in the four-dimensional limit $\beta \rightarrow 0$, the right hand side of (3.8) starts as $z^{2}$, so all correlators with $J<2$ receive no instanton corrections. The first non-trivial correlator is then given by the coefficient of the term $z^{2}$ in (3.8)

$$
\left\langle\operatorname{tr} \phi^{2}\right\rangle=\operatorname{tr} a^{2}-\frac{2 \epsilon_{1} \epsilon_{2}}{Z_{4 d}} \sum_{Y} q^{k} k Z_{4 k}=\operatorname{tr} a^{2}-2 q \frac{d}{d q} \mathcal{F}_{4}(q)=\operatorname{tr} a^{2}-2 \mu\left(\epsilon_{1}+\epsilon_{2}\right) \frac{q}{1-q}
$$

Remarkably, as in the eight dimensional case, the chiral correlators of the orbifold theory, at the leading order in the $\Omega$ background, can be represented in a closed form. The result can be written as

$$
\left\langle\operatorname{tr} \frac{1}{x-\phi}\right\rangle=\frac{P^{\prime}(x)}{P(x)}+\left(\epsilon_{1}+\epsilon_{2}\right) \frac{\partial^{2}}{\partial x^{2}}\left[\log \left(\frac{1-q}{(1-q R(x))}\right)+O(\epsilon)\right],
$$

where $P(x), R(x)$ are given again by (2.31). One finds again exotic instanton corrections to the correlators in the $\mathrm{SU}(\mathrm{N})$ theory. For example for the $\mathrm{SU}(2)$ theory, with two flavours and $a_{1}=-a_{2}=a, m_{1}=-m_{2}=m$, the first non-trivial correlators are

$$
\begin{aligned}
& \left\langle\operatorname{tr} \phi^{3}\right\rangle=6\left(\epsilon_{1}+\epsilon_{2}\right)\left(a^{2}-m^{2}\right)\left(q+q^{2}+q^{3}+\ldots\right) \\
& \left\langle\operatorname{tr} \phi^{5}\right\rangle=10\left(\epsilon_{1}+\epsilon_{2}\right)\left(a^{2}-m^{2}\right)\left(2 a^{2} q+\left(3 a^{2}-m^{2}\right) q^{2}+2\left(2 a^{2}-m^{2}\right) q^{3}+\ldots\right)
\end{aligned}
$$

and so on. 


\section{Acknowledgments}

R. Poghossian wants to thank the I.N.F.N. unit Tor Vergata for hospitality and financial support during the completion of this work. He also acknowledges the support in the framework of Armenian SCS grant 20RF-142.

\section{A Transformation properties of the function $D_{s}(\tau)$}

In this appendix we study the transformation properties of the series

$$
D_{s}(\tau)=\sum_{n=1}^{\infty} \sigma_{s-1}(n) q^{n}=\sum_{n=1}^{\infty} \frac{n^{s-1} q^{n}}{1-q^{n}}
$$

where $\sigma_{s}(n)=\sum_{d \mid n} d^{s}$ is the sum over the positive divisor function, under the modular group

$$
\tau \rightarrow \frac{a \tau+b}{c \tau+d}, \quad\left(\begin{array}{ll}
a & b \\
c & d
\end{array}\right) \in \mathrm{SL}(2, \mathbb{Z})
$$

An integral formula for these transformations is given in [29]

$$
\begin{aligned}
D_{3}\left(\frac{a \tau+b}{c \tau+d}\right)= & (c \tau+d)^{s}\left[D_{s}(\tau)+\frac{1}{2}\left(1-(c \tau+d)^{-s} \zeta(3)\right)\right. \\
& \left.+\frac{c^{s-1} \sec \frac{\pi s}{2}}{8(c \tau+d)^{s / 2}} \int_{C}(-z)^{s-1}\left(c+\sum_{j=0}^{c-1} \cot \pi\left(i \sqrt{c \tau+d} z-\frac{j d}{c}\right) \cot \pi\left(\frac{i z}{\sqrt{c \tau+d}}-\frac{j}{c}\right)\right) d z\right]
\end{aligned}
$$

where $\mathrm{C}$ is the Hankel contour encircling the positive real axis in the clockwise direction but not other poles of the integrand. From its definition (A.1), it is clear that $D_{s}(\tau)$ is invariant under $\mathrm{T}: \tau \rightarrow \tau+1$ transformations. The $\mathrm{S}: \tau \rightarrow-1 / \tau$ transformation yields

$$
\begin{aligned}
D_{s}\left(-\frac{1}{\tau}\right)= & \tau^{s}\left(D_{s}(\tau)+\frac{1}{2}\left(1-\tau^{-s} \zeta(s-1)\right)\right. \\
& \left.+\frac{1}{8 \tau^{s / 2} \cos (\pi s / 2)} \int_{C}(-z)^{s-1}\left(1+\cot (\pi i \sqrt{\tau} z) \cot \frac{\pi i z}{\sqrt{\tau}}\right) d z\right)
\end{aligned}
$$

For $s=3$ the second line in (A.3) is an indeterminate form. Using L'Hopital rule we get

$$
\frac{1}{4 \pi \tau^{3 / 2}} \int_{C} z^{2} \log (-z)\left(1+\cot (\pi i \sqrt{\tau} z) \cot \frac{\pi i z}{\sqrt{\tau}}\right) d z
$$

The values of logarithm along the upper and lower branches of the contour $C$ differ by $2 \pi i$, so the contour integral (A.4) can be rewritten as an ordinary integral

$$
\frac{i}{2 \tau^{3 / 2}} \int_{0}^{\infty} x^{2}\left(1+\cot (\pi i \sqrt{\tau} x) \cot \frac{\pi i x}{\sqrt{\tau}}\right) d x
$$

or equivalently

$$
-\frac{i}{\tau^{3 / 2}} \int_{0}^{\infty} x^{2} \frac{e^{-\frac{2 \pi x}{\sqrt{\tau}}}+e^{-2 \pi \sqrt{\tau} x}}{\left(1-e^{-\frac{2 \pi x}{\sqrt{\tau}}}\right)\left(1-e^{-2 \pi \sqrt{\tau} x}\right)} d x
$$


Expanding the denominator as a double geometric series and performing the elementary integration we obtain

$$
\frac{i}{2 \pi^{3}}\left(\sum_{m, n=1}^{\infty} \frac{1}{(m \tau+n)^{3}}+\frac{1+\tau^{-3}}{2} \zeta(3)\right)
$$

leading to

$$
\tau^{-3} D_{3}(-1 / \tau)=D_{3}(\tau)+\frac{i}{2 \pi^{3}}\left(\sum_{m, n=1}^{\infty} \frac{1}{(m \tau+n)^{3}}+\frac{1+\tau^{-3}}{2} \zeta(3)\right)
$$

which already appeared in [30]. An equivalent formula for the S-transformation can be obtained by using the T-invariance and considering the modular transformation

$$
\text { TST }: \quad \tau \rightarrow-\frac{1}{\tau+1}+1=-\frac{1}{-1-\tau^{-1}}
$$

One finds

$$
\begin{aligned}
-\tau^{-3} D_{3}(-1 / \tau)= & D_{3}(\tau) \\
& +\frac{i}{2 \pi^{3}}\left(\sum_{m, n=1}^{\infty} \frac{1}{(m(\tau+1)+n)^{3}}+\frac{1}{(-m(\tau+1)+n \tau)^{3}}+\frac{1+\tau^{-3}}{2} \zeta(3)\right)
\end{aligned}
$$

Summing (A.8) with (A.10) we see that the l.h.s.'s cancel and the double sums and $\zeta(3)$ nicely combine into

$$
D_{3}(\tau)=-\frac{i}{4 \pi^{3}} \sum_{m=1}^{\infty} \sum_{n=-\infty}^{\infty} \frac{1}{(m \tau+n)^{3}}
$$

Similar manipulations lead to the general double sum representation [30]

$$
D_{s}(\tau)=\frac{e^{\pi i s / 2} \Gamma(s)}{(2 \pi)^{s}} \sum_{m=1}^{\infty} \sum_{n=-\infty}^{\infty} \frac{1}{(m \tau+n)^{s}}
$$

from which it follows

$$
\begin{aligned}
D_{s}\left(\frac{a \tau+b}{c \tau+d}\right) & =\frac{e^{\pi i s / 2} \Gamma(s)}{(2 \pi)^{s}} \sum_{m=1}^{\infty} \sum_{n=-\infty}^{\infty} \frac{(c \tau+d)^{s}}{(m(a \tau+b)+n(c \tau+d))^{s}} \\
& =\frac{e^{\pi i s / 2} \Gamma(s)(c \tau+d)^{s}}{(2 \pi)^{s}} \sum_{m=1}^{\infty} \sum_{n=-\infty}^{\infty} \frac{1}{((m a+n c) \tau+(m b+n d))^{s}} \\
& =\frac{e^{\pi i s / 2} \Gamma(s)(c \tau+d)^{s}}{(2 \pi)^{s}} \sum_{(\tilde{n}, \tilde{m}) \in \Omega} \frac{1}{(\tilde{m} \tau+\tilde{n})^{s}}
\end{aligned}
$$

The sum in the last line runs over the points above the line $L=\{(n, m) \mid c n-d m=0\}$ (the red line in figure 1). Comparing against the sum in (A.12) running over points in the upper half plane one finds that in the difference

$$
(c \tau+d)^{-s} D_{s}\left(\frac{a \tau+b}{c \tau+d}\right)-D_{s}(\tau)
$$




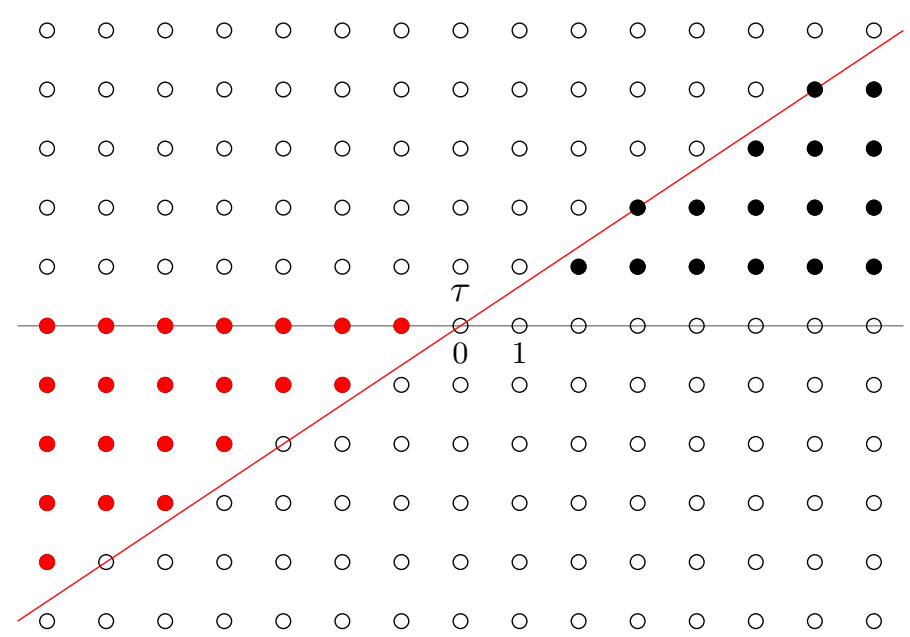

Figure 1. Summation regions. The case $a=3, b=4, c=2, d=3$.

all the white dots in figure 1 cancel and one is left with a sum over the red dots minus a sum over the black dots. Now it becomes obvious the crucial difference between the cases with even and odd values of $s$. In the former case the contributions of the red and black dots (besides those which lie on the black and red lines respectively) completely cancel out and we get

$$
(c \tau+d)^{-s}\left(\frac{e^{\pi i s / 2} \Gamma(s) \zeta(s)}{(2 \pi)^{s}}+D_{s}\left(\frac{a \tau+b}{c \tau+d}\right)\right)=\frac{e^{\pi i s / 2} \Gamma(s) \zeta(s)}{(2 \pi)^{s}}+D_{s}(\tau)
$$

where the $\zeta$ functions are just the contributions of the dots lying on the black and red lines. As a result the combination

$$
\frac{e^{\pi i s / 2} \Gamma(s) \zeta(s)}{(2 \pi)^{s}}+D_{s}(\tau)
$$

is a weight $s$ modular form. Instead for odd $s$ we get twice the contributions of the inner red boxes (those lying strictly below the black line) together with the difference of the contributions of the red dots lying on the black line and the black dots lying on the red line. The final result now reads:

$$
\begin{aligned}
(c \tau+d)^{-s} D_{s}\left(\frac{a \tau+b}{c \tau+d}\right)= & D_{s}(\tau) \\
& -\frac{e^{\pi i s / 2} \Gamma(s)}{(2 \pi)^{s}}\left(\left(1+(c \tau+d)^{-s}\right) \zeta(s)+2 \sum_{m=1}^{\infty} \sum_{n=1}^{\infty} \frac{1}{((c \tau+d) m+n)^{s}}\right)
\end{aligned}
$$

In particular for $s=3$ we find the transformation (2.33). 
Open Access. This article is distributed under the terms of the Creative Commons Attribution License (CC-BY 4.0), which permits any use, distribution and reproduction in any medium, provided the original author(s) and source are credited.

\section{References}

[1] E. Witten, Bound states of strings and p-branes, Nucl. Phys. B 460 (1996) 335 [hep-th/9510135] [INSPIRE].

[2] M.R. Douglas, Gauge fields and D-branes, J. Geom. Phys. 28 (1998) 255 [hep-th/9604198] [INSPIRE].

[3] M.B. Green and M. Gutperle, D Particle bound states and the D instanton measure, JHEP 01 (1998) 005 [hep-th/9711107] [INSPIRE].

[4] M.B. Green and M. Gutperle, D instanton partition functions, Phys. Rev. D 58 (1998) 046007 [hep-th/9804123] [INSPIRE].

[5] M.B. Green and M. Gutperle, D instanton induced interactions on a D3-brane, JHEP 02 (2000) 014 [hep-th/0002011] [INSPIRE].

[6] M. Billó, M. Frau, I. Pesando, F. Fucito, A. Lerda and A. Liccardo, Classical gauge instantons from open strings, JHEP 02 (2003) 045 [hep-th/0211250] [INSPIRE].

[7] R. Blumenhagen, M. Cvetič and T. Weigand, Spacetime instanton corrections in $4 D$ string vacua: The Seesaw mechanism for D-brane models, Nucl. Phys. B 771 (2007) 113 [hep-th/0609191] [INSPIRE].

[8] L.E. Ibáñez and A.M. Uranga, Neutrino Majorana Masses from String Theory Instanton Effects, JHEP 03 (2007) 052 [hep-th/0609213] [INSPIRE].

[9] R. Argurio, M. Bertolini, S. Franco and S. Kachru, Gauge/gravity duality and meta-stable dynamical supersymmetry breaking, JHEP 01 (2007) 083 [hep-th/0610212] [INSPIRE].

[10] R. Argurio, M. Bertolini, G. Ferretti, A. Lerda and C. Petersson, Stringy instantons at orbifold singularities, JHEP 06 (2007) 067 [arXiv:0704.0262] [INSPIRE].

[11] M. Bianchi, F. Fucito and J.F. Morales, D-brane instantons on the $T^{6} / Z_{3}$ orientifold, JHEP 07 (2007) 038 [arXiv: 0704.0784] [INSPIRE].

[12] R. Blumenhagen, M. Cvetič, D. Lüst, R. Richter and T. Weigand, Non-perturbative Yukawa Couplings from String Instantons, Phys. Rev. Lett. 100 (2008) 061602 [arXiv:0707.1871] [INSPIRE].

[13] L.E. Ibáñez, A.N. Schellekens and A.M. Uranga, Instanton Induced Neutrino Majorana Masses in CFT Orientifolds with MSSM-like spectra, JHEP 06 (2007) 011 [arXiv:0704 .1079] [INSPIRE].

[14] L.E. Ibáñez and A.M. Uranga, Instanton induced open string superpotentials and branes at singularities, JHEP 02 (2008) 103 [arXiv:0711.1316] [INSPIRE].

[15] M. Billó, L. Gallot, A. Lerda and I. Pesando, F-theoretic versus microscopic description of a conformal $N=2 S Y M$ theory, JHEP 11 (2010) 041 [arXiv: 1008.5240] [INSPIRE].

[16] F. Fucito, J.F. Morales and D.R. Pacifici, Multi instanton tests of holography, JHEP 09 (2011) 120 [arXiv:1106.3526] [INSPIRE].

[17] N. Nekrasov, Magnificent Four, arXiv:1712.08128 [INSPIRE]. 
[18] N. Nekrasov and N. Piazzalunga, Magnificent Four with Colors, Commun. Math. Phys. 372 (2019) 573 [arXiv : 1808.05206] [inSPIRE].

[19] M. Billo, M. Frau, F. Fucito, L. Gallot, A. Lerda and J. Morales, On the D(-1)-D7 brane system, in preparation (2020).

[20] F. Fucito, J.F. Morales and R. Poghossian, Instantons on quivers and orientifolds, JHEP 10 (2004) 037 [hep-th/0408090] [INSPIRE].

[21] M. Billó, M. Frau, F. Fucito, A. Lerda, J.F. Morales and R. Poghossian, Stringy instanton corrections to $N=2$ gauge couplings, JHEP 05 (2010) 107 [arXiv:1002.4322] [INSPIRE].

[22] S.K. Donaldson, Anti-self-dual Yang-Mills connexions over complex algebraic surfaces and stable vector bundles, Proc. Lond. Math. Soc. s3-50 (1) (1985) 1.

[23] R. Flume and R. Poghossian, An Algorithm for the microscopic evaluation of the coefficients of the Seiberg-Witten prepotential, Int. J. Mod. Phys. A 18 (2003) 2541 [hep-th/0208176] [INSPIRE].

[24] U. Bruzzo, F. Fucito, J.F. Morales and A. Tanzini, Multiinstanton calculus and equivariant cohomology, JHEP 05 (2003) 054 [hep-th/0211108] [INSPIRE].

[25] A.S. Losev, A. Marshakov and N.A. Nekrasov, Small instantons, little strings and free fermions, in From Fields to Strings: Circumnavigating Theoretical Physics: A Conference in Tribute to Ian Kogan, (2003) [hep-th/0302191] [INSPIRE].

[26] R. Flume, F. Fucito, J.F. Morales and R. Poghossian, Matone's relation in the presence of gravitational couplings, JHEP 04 (2004) 008 [hep-th/0403057] [INSPIRE].

[27] F. Fucito, J.F. Morales and R. Poghossian, Exotic prepotentials from D(-1)D"7 dynamics, JHEP 10 (2009) 041 [arXiv: 0906.3802] [INSPIRE].

[28] N. Nekrasov and A. Okounkov, Seiberg-Witten theory and random partitions, Prog. Math. 244 (2006) 525 [hep-th/0306238] [INSPIRE].

[29] N. Kim, Transformations of some Lambert Series and Cotangent Sums, Mathematics 7 (2019) 840.

[30] S. Shimomura, Modularity Gap for Eisenstein Series, Proc. Japan Acad. 86 (2010) 79. 